University of Wollongong

Research Online

Faculty of Engineering and Information

Faculty of Engineering and Information

Sciences - Papers: Part B

Sciences

2010

HRP-2 plays the yoyo: From human to humanoid yoyo playing using optimal control

Katja Mombaur

University of Heidelberg

Manish Sreenivasa

University of Wollongong, manishs@uow.edu.au

Follow this and additional works at: https://ro.uow.edu.au/eispapers1

Part of the Engineering Commons, and the Science and Technology Studies Commons

Research Online is the open access institutional repository for the University of Wollongong. For further information contact the UOW Library: research-pubs@uow.edu.au 


\title{
HRP-2 plays the yoyo: From human to humanoid yoyo playing using optimal control
}

\begin{abstract}
Yoyo playing may seem easy for a human, but it is a challenging problem for a humanoid robot. This paper presents an approach to generate yoyo motions for the humanoid robot, HRP-2, based on motion recorded from human yoyo playing, dynamical models and numerical optimal control techniques. We recorded vertical yoyo playing of 4 subjects measuring yoyo height and rotation angle as well as the corresponding hand motions. A detailed multi-phase yoyo model with impact collisions and control patterns of human yoyo playing were identified from these measurements. Based on this knowledge, reliable yoyo motions within the feasibility ranges of HRP-2 were generated using optimal control. The resulting motions have been implemented on the robot using open-loop and event-based quasi open-loop control strategies.
\end{abstract}

\section{Keywords}

optimal, playing, yoyo, humanoid, human, yoyo:, plays, control, hrp-2

Disciplines

Engineering | Science and Technology Studies

\section{Publication Details}

Mombaur, K. \& Sreenivasa, M. N. (2010). HRP-2 plays the yoyo: From human to humanoid yoyo playing using optimal control. 2010 IEEE International Conference on Robotics and Automation (pp. 3369-3376). United States: IEEE. 


\title{
HRP-2 plays the yoyo: From human to humanoid yoyo playing using optimal control
}

\author{
Katja Mombaur and Manish N. Sreenivasa
}

\begin{abstract}
Yoyo playing may seem easy for a human, but it is a challenging problem for a humanoid robot. This paper presents an approach to generate yoyo motions for the humanoid robot, HRP-2, based on motion recorded from human yoyo playing, dynamical models and numerical optimal control techniques. We recorded vertical yoyo playing of 4 subjects measuring yoyo height and rotation angle as well as the corresponding hand motions. A detailed multi-phase yoyo model with impact collisions and control patterns of human yoyo playing were identified from these measurements. Based on this knowledge, reliable yoyo motions within the feasibility ranges of HRP-2 were generated using optimal control. The resulting motions have been implemented on the robot using open-loop and event-based quasi open-loop control strategies.
\end{abstract}

\section{INTRODUCTION}

As children (and even some adults) many of us have played with a yoyo for fun. While this does not seem to be a complicated task to learn, in reality, manipulating a yoyo involves a complex interplay between sensing its position, estimating its future behavior and co-ordinating one's motor action to compensate for perturbations and maintaining stable oscillations. Keeping this in mind, yoyo playing for a humanoid robot is a challenging task for several reasons. First, compared to a human, humanoid robots are relatively slow, capable of only small accelerations and a limited range of motion. Hence, a direct transfer of human maneuvers by simply replaying the recorded motion on a humanoid would not work. Second, the sensors and controllers available even on a state-of-the-art humanoid robot are quite different from what nature has offered humans. And third, there is little formal understanding of the basic control laws that humans apply to perform cyclic tasks such as yoyo playing including the amount and type of feedback.

In this paper we establish a link between human and humanoid yoyo playing using motion capture experiments, dynamical models of yoyo motions and optimal control techniques. Our goal is to compute trajectories and control inputs that enable the humanoid robot HRP-2 to perform cyclic yoyo playing motions.

There are other cyclic tasks involving the manipulation of a ball such as juggling and dribbling which have received a lot of attention in robotics [1], [2], [3]. The fundamental difference between ball manipulation and yoyo playing is that juggling and dribbling have free flying phases where

This work has been supported by the French ANR project LOCANTHROPE

Katja Mombaur and Manish N. Sreenivasa are with LAAS-CNRS, 7 Avenue du Colonel Roche, 31077 Toulouse, France (phone: +33 (0)5 613379 02; fax: +33 (0)5 613364 55);kmombaurelaas.fr, manuelaas.fr

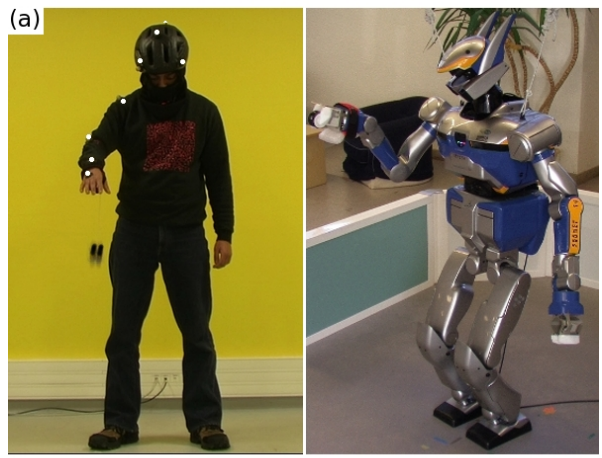

(b)

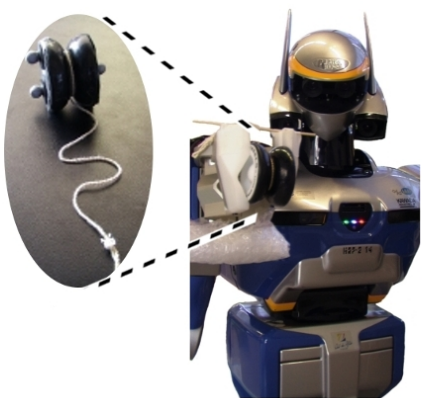

Fig. 1. (a) Human yoyo playing motion recorded via motion capture technology (left), is used to teach the humanoid HRP-2 (right) (b) HRP2 grasps the yoyo in its hand, and inset shows the same yoyo with the reflective motion capture markers used in the motion capture experiments

the ball and the hand move independently, while the yoyo always remains connected to the hand by the string.

Several authors have studied yoyo playing from a robotics perspective. Hashimoto and Noritsugu [4], proposed a model to control a yoyo by a robot based on vision feedback. An improved model with 2 DoF (Degrees of Freedom) and four phases was used by Jin and Zacksenhouse [5] to study energy loss due to collisions. The authors later proposed a switching control strategy and use visual feedback and state estimation to implement yoyo motion on a manipulator [6]. Yoyo motions of different amplitudes were implemented on a robotic arm, using a 1 DoF yoyo model, by Zlajpah and Nemec [7].

One of the contributions of this paper is to attempt, for the first time, to teach yoyo playing to a humanoid robot and not a robotics arm. A humanoid robot is not only a much more complex kinematic structure but also has unique characteristics that are both a constraint and an advantage to its overall capabilities. For example, a typical humanoid is not capable of the kind of precise high-speed motion that robotics arms can accomplish. In addition, a humanoid, 


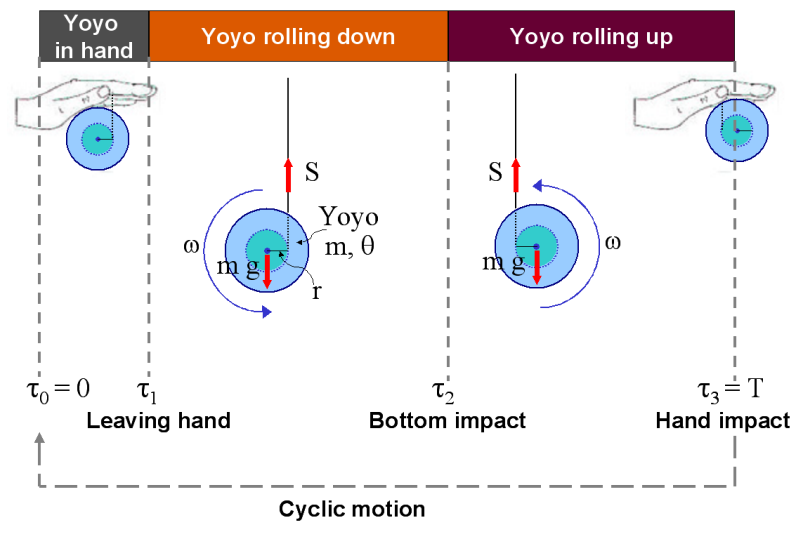

Fig. 2. Different phases of yoyo play: (1) yoyo in hand, (2) yoyo rolling down, (3) yoyo rolling up

being bipedal, has to maintain its stability while executing all motions and for the same reason cannot execute the nonsmooth control laws proposed by previous authors.

An important aspect of our work is the detailed study of human yoyo playing motion. The foundation of our yoyo model on human motion capture data enables us to develop a very precise model of its behavior. Another contribution of this paper is the use of optimal control techniques in the identification of human motions and for the generation of humanoid yoyo motion.

Our work is also different with respect to the type of yoyo used. Previous studies on this topic have worked with and modeled "fixed-axis" yoyos. In this kind of yoyo the string is fixed to the axis which makes the yoyo return a part of the way even if the guiding hand is not doing anything. In contrast to this, we work with the more complex but also more versatile "free-wheeling" yoyo type which allows the yoyo to rotate freely at the end of the string (a maneuver which is called a "sleeper"). It is important to note that a free-wheeling yoyo will only return if the hand is sufficiently accelerated at the right time.

The motion capture experiments that we have performed include a variety of 3D yoyo motions. In this paper, we restrict ourselves to the analysis and experimental implementation of up-and-down yoyo motion.

The remainder of the paper is organized as follows. Section II summarizes the motion capture experiments of human yoyo playing. In section III, we present the dynamic yoyo model that we use for our computations. In section IV we show, how the model can reproduce experimental trajectories by identifying the correct hand accelerations and setting some unknown parameters by optimal control techniques. Section V describes how, again using optimal control, feasible yoyo playing trajectories can be generated for the robot HRP-2. In section VI, we present experimental results on the humanoid robot HRP-2.

\section{MEASURING HUMAN YOYO MOTION}

Human yoyo playing was recorded using the aid of motion capture technology. The experimental area was a $5 \times 5 \mathrm{~m}$ well lit empty space equipped with an optical tracking system (Motion Analysis Corporation, USA). The tracking system consists of 10 infra-red cameras, which allow for an accuracy of position tracking of better than $1 \mathrm{~mm}$ in the entire capture area. Sampling rate was $100 \mathrm{~Hz}$ and all data were output in a reference frame centered in the capture volume. The yoyo used was a professional grade free-wheeling type (Duncan Wheels, Duncan, USA) weighing 66 gram. Three miniature reflective tracking markers were attached to the yoyo in order to track its trajectory during the oscillations (see Fig. 1-b). Two markers were attached symmetrically to one side of the yoyo, and the third marker was set in the middle of the other side. Marker positions were adjusted such that they caused minimal perturbation during oscillations.

To track the movement of the human, we used 8 tracking markers as shown in Fig. 1-a. In total we measured yoyo action of four participants, all of whom had previous experience with playing yoyos. All participants chose to use their right hand and were free to choose the finger on which to tie the yoyo string. They were asked to elevate their hand, instead of just lifting the finger, while playing the yoyo. This was necessary due to the lack of finger articulation on our humanoid robot HRP-2.

Participants were instructed to play the yoyo at their preferred speed in a smooth fashion, and to not re-grasp the yoyo between trials. Before recording all participants were given about 5 minutes to practice yoyo oscillations with the given instructions. None of the participants reported any difficulty playing with the yoyo in this manner. For each participant, we recorded three trials, each containing 90 seconds of up-down yoyo motion.

\section{A DYNAMIC YOYO MODEL}

Even though a yoyo seems to be a quite simple mechanism, the correct description of its dynamics is not that straightforward. A complete description involves multiple phases, state dependent phase switching events, discontinuities of variables at impacts, friction effects etc. The yoyo motion is powered by the motion of the guiding hand which transfers energy to the yoyo either by directly imposing its motion to the yoyo during the short contact phase, or - when the yoyo has left the hand - by an indirect transfer of hand translation via the string into yoyo translation as well as rotation. The fact that the string provides a constant link between the hand and the manipulated object, makes a yoyo model fundamentally different from that of a ball dribbling or paddle juggling task.

A yoyo has six degrees of freedom - three translational and three rotational - and there are various effects that can make the direction of its axis of rotation change. In this paper we consider only standard up-and-down yoyo-playing (other maneuvers which also have been measured will be modeled in a future paper). For this type of motion it can be seen from measurements, that,

- the yoyo is essentially moving along a vertical line (in $z$ direction); 
- the axis of rotation of a yoyo generally keeps constant for one up-and-down cycle (it may slightly change between cycles due to interference of the hand).

For this purpose, we have developed a model of cyclic vertical yoyo playing of a free-wheeling yoyo which contains the different phases and impact events shown in Fig. 2. From our motion capture data we gain information about the string length (via hand and yoyo positions) and of the rotational state of the yoyo at each time which will help us determine the free parameters of the model.

For the description of the yoyo motion, we use seven state variables, namely the position variables $h, z$, and $\phi$ which are the hand height, yoyo height, and yoyo rotational angle, as well as the corresponding velocities $v_{h}=\dot{h}, v_{z}=\dot{z}$, and $\omega=\dot{\phi}$, and the radius $r$. The radial distance $r$ at which the string acts on the yoyo is actually the combination of the rigid yoyo axis and of the layers of the wound-up part of the string. Previous authors have either worked with a constant [5] or linear [7] approximation of this radial dependency on the string length. Since the radius is important to characterize the relation between translational and rotational yoyo motion, we have decided to model it more precisely and include it as a state variable of the system. The height variable $h$ is defined to be zero at the beginning of the cycle. The acceleration of the hand $a_{h}$ is considered to be the guiding input variable (also called control variable) of the yoyo system. The rotational angle of the yoyo $\phi$ is defined to be zero at the beginning, and the sense of rotation is defined to be positive throughout - note that the sense of rotation does not change during one cycle (starting and ending when the yoyo touches the hand). The yoyo string length is an important quantity. By $l_{\max }$, we denote the total length of the string which at every instant $t$ can be decomposed into the currently active string length $l$ and the wound-up part of the string $l_{i}$, i.e. $l_{\max }=l(t)+l_{i}(t) . l(t)$ is defined to be zero when the yoyo is at the closest possible distance to the hand, and equal to $l_{\max }$ when the string is completely unwound.

In what we define to be the beginning of the cycle there is a very short phase in which the yoyo is in close contact with the hand - i.e. it has the same translational velocity as the hand - and does not rotate. The motion of this system follows the equations:

$$
\begin{aligned}
\dot{h}=\dot{z} & =v_{h} \\
\dot{\phi} & =0
\end{aligned}
$$

which in the full form with seven state variables becomes:

$$
\begin{aligned}
\dot{h} & =v_{h} \\
\dot{z} & =v_{z} \\
\dot{\phi} & =\omega \\
\dot{v_{h}} & =a_{h} \\
\dot{v}_{z} & =a_{h} \\
\dot{\omega} & =0 \\
\dot{r} & =0
\end{aligned}
$$

with the initial conditions:

$$
\begin{aligned}
z\left(\tau_{0}\right) & =h\left(\tau_{0}\right)+\Delta_{h} \\
v_{z}\left(\tau_{0}\right) & =v_{h}\left(\tau_{0}\right) \\
\phi\left(\tau_{0}\right) & =\omega\left(\tau_{0}\right)=0 .
\end{aligned}
$$

$\Delta_{h}$ is the small offset between hand and yoyo mid point, occurring when the two are at close contact.

After a very short time (which was estimated to be $t=$ $\tau_{1}=0.1 \mathrm{~s}$ ) the yoyo leaves the hand and starts its rolling down phase. In this phase, (3) - (6) remain valid, but the last three equations change. The rolling motion induces the following kinematic coupling to the velocity variables $v_{h}, v_{z}$ and $\omega$ :

$$
\begin{aligned}
\dot{l} & =v_{h}-v_{z}=\omega r \\
\rightarrow \ddot{l} & =\dot{v}_{h}-\dot{v}_{z}=\omega \dot{r}+\dot{\omega} r \\
\rightarrow \dot{v}_{z}+\dot{\omega} r & =a_{h}-\omega \dot{r}
\end{aligned}
$$

The dynamic equations of the yoyo in translational and rotational directions can be written as:

$$
\begin{aligned}
m \dot{v}_{z} & =S-m g \\
\Theta \dot{\omega} & =S r-\gamma r \omega
\end{aligned}
$$

where $m$ and $\Theta$ are the mass and moment of inertia of the yoyo. $S$ is the force in the string (see Fig. 2), and the last term in the latter equation is a velocity dependent friction term with constant $\gamma$. Elimination of $S$ results in the dynamic equation:

$$
\Theta \dot{\omega}-m r \dot{v}_{z}=m g r-\gamma r \omega
$$

For the radial distance at which the string acts on the yoyo we can assume that its change is proportional to the angular velocity of the yoyo (due to the snail-like way the string winds about the axis). In the rolling down phase, the radius decreases, and the angular velocity by definition is positive. We therefore obtain:

$$
\dot{r}=-\rho \omega
$$

where the parameter $\rho>0$ remains to be determined.

The rolling down phase lasts until the bottom impact event at $t=\tau_{2}$ when the string reaches its maximum length,

$$
s_{\text {bottom impact }}=l-l_{\max }=h-z-\Delta_{h}-l_{\max }=0
$$

i.e. obviously this event does not explicitly depend on time, but on the state of the yoyo system. It can be assumed that the motion of the hand is smooth, but the impact generates discontinuities of the velocities of the yoyo $v_{z}$ and $\omega$ of the following form:

$$
\begin{aligned}
\omega\left(\tau_{2}^{+}\right) & =\beta \omega\left(\tau_{2}^{-}\right) \quad \text { with } \beta<1 \\
v_{z}\left(\tau_{2}^{+}\right) & =\left\{\begin{array}{l}
v_{h}\left(\tau_{2}^{+}\right)+\omega\left(\tau_{2}^{+}\right) r \text { if }\left(a_{h}\left(\tau_{2}^{-}\right) \geq b_{a_{h}}\right) \\
v_{h}\left(\tau_{2}^{+}\right)
\end{array}\right.
\end{aligned}
$$

Equation (17) assumes that the loss of rotational motion can be captured by a constant factor $\beta$ (to be determined from measurements). Equation. (18) takes into account the property of a free-wheeling yoyo that if no sufficient upward 
acceleration is generated before bottom impact, then it will remain in a freely rotating - i.e. sleeping - mode at the end of the string. If on the other hand the upward acceleration is high enough, the yoyo will go into the up-winding phase. It is this latter case that we are interested in for the purpose of this paper. The lower bound of this acceleration depends on many factors, such as the tightness of the string about the yoyo axle which also changes during the play, but we work here with an estimation of $b_{a_{h}}=3 \mathrm{~m} / \mathrm{s}^{2}$. With this estimation we remain on the safe side for motion generation, since we observed that humans never had to exceed that value at the bottom point.

The rolling up phase is defined by similar equations as the rolling down phase but with some sign changes of the terms. The sense of rotation remains unchanged (positive), but the active string length now shortens as a function of the rotation, i.e. the kinematic equation becomes

$$
\begin{aligned}
\dot{l} & =v_{h}-v_{z}=-\omega r \\
\rightarrow \dot{v}_{z}-\dot{\omega} r & =a_{h}+\omega \dot{r}
\end{aligned}
$$

The dynamic equation also changes to,

$$
\Theta \dot{\omega}+m r \dot{v}_{z}=-m g r-\gamma r \omega,
$$

and the radius equation becomes

$$
\dot{r}=\rho \omega .
$$

Again, (3) - (6) remain valid.

The phase ends when the yoyo is back in the hand at $t=\tau_{3}=T$ which is implicitly defined by the switching function

$$
s_{\text {hand impact }}=l=h-z-\Delta_{h}=0,
$$

and the cycle is closed by appropriate impact equations to match the initial conditions (10) - (12). Periodicity constraints are considered to generate a cyclic motion:

$$
\left(h(T), z(T), v_{h}(T), r(T)\right)=\left(h(0), z(0), v_{h}(0), r(0)\right)
$$

Periodicity of $v_{z}$ is automatically implied via (23) and (11), and periodicity of $\omega$ via the hand impact equations that eliminate all rotation of the yoyo. $\phi$ is by definition reset to zero after each cycle.

For the yoyo used in the human experiments, only the yoyo mass could be directly measured, and was $m=0.066 \mathrm{~kg}$. The inertia $\Theta$ of the yoyo can only be roughly estimated and is identified by computations. The same is true for the impact coefficient $\beta$, the friction coefficient $\gamma$ and the radial parameter $\rho$. Maximum string length $l_{\max }$ and yoyo-hand offset depend on the way the yoyo is attached to the hand of each subject and will also be identified from measurements (see next section).

Jin and Zacksenhouse [5] have proposed the inclusion of an additional transition phase after bottom impact where no rolling occurs and $z$ and $\phi$ are independent. However, for the type of yoyo used here we determined from motion capture that such a phase is not present, and that it would not improve the fit if $z$ and $\phi$ were allowed to change independently. For this free-wheeling type of yoyo all bottom impact events can be summarized in the impact model described above.

\section{IDENTIFICATION OF HUMAN YOYO CONTROL SCHEME}

Based on the yoyo model described in the previous section, the control scheme - i.e. the hand acceleration pattern that the subject is using - can be identified, along with the unknown parameters. In order to do so, we have formulated and solved a multi-phase optimal control problem with a least-squares objective function of the following form (which can also be called an identification problem):

$$
\begin{aligned}
\min _{x(\cdot), u(\cdot), p} & \sum_{k=1}^{m}\left\|W\left(x_{\text {pos }}\left(t_{k}\right)-\hat{x}_{\text {pos }}\left(t_{k}\right)\right)\right\|_{2}^{2} \\
\text { s. t. } \dot{x}(t)= & f_{j}(t, x(t), u(t), p) \quad \text { for } t \in\left[\tau_{j-1}, \tau_{j}\right] \\
& j=1, \ldots, 3, \tau_{0}=0, \tau_{3}=T \\
x\left(\tau_{j}^{+}\right)= & \left.x\left(\tau_{j}^{-}\right)\right)+J\left(\tau_{j}^{-}\right) \quad \text { for } j=1, \ldots, 3 \\
0= & r_{e q}(x(0), . ., x(T), p) \\
0 \leq & r_{i n e q}(x(0), . ., x(T), p) \\
0 \leq & g_{j}(t, x(t), u(t), p) \quad \text { for } t \in\left[\tau_{j-1}, \tau_{j}\right]
\end{aligned}
$$

In this formulation, $x$ are the state variables of this problem with $x^{T}=\left(h, z, \phi, v_{h}, v_{z}, \omega, r\right)$. In the objective function (24), we use that subset of state variables that can be measured, namely the yoyo positions $x_{p o s}^{T}=(h, z, \phi)$. The least squares term minimizes the squared distance of the computed values of $x_{\text {pos }}$ from the measured values $\hat{x}_{\text {pos }}$ at $m$ measurement points $t_{k}$, using an appropriate diagonal scaling matrix $W$ to adjust variables to the same order of magnitude. $u=\left(a_{h}\right)$ is the vector of control variables and $p^{T}=$ $\left(\Theta, \beta, \gamma, \rho, \Delta_{h}, l_{\max }\right)$ the vector of free model parameters. $\tau_{j}$ are the phase switching points as defined in section II. This problem formulation includes constraints of different types. Equation (25) represents the dynamic model equations for phases $1-3$, and (26) the state variable jumps between those. Equations (27) and (28) are pointwise equality and inequality constraints. They may describe conditions to a single point, such as starting equation (10) or switching point conditions equation (16) and (22), or conditions relating different points to each other, such as periodicity conditions (23). Equation (29) represents continuous inequality constraints on all variables, such as bounds on state and control variables, which are not relevant in this section, since they are not reached in the case of human yoyo playing, but they become relevant in the next section where humanoid yoyo playing is considered.

For the solution of this multi-phase optimal control problem, we use a direct approach based on a piecewise constant control discretization as implemented in the code MUSCOD ([8], [9]). State parameterization is accomplished by the multiple shooting method which splits the long integration interval into many smaller ones and with that transforms the original boundary value problem into a set of initial value problems with corresponding continuity and boundary 

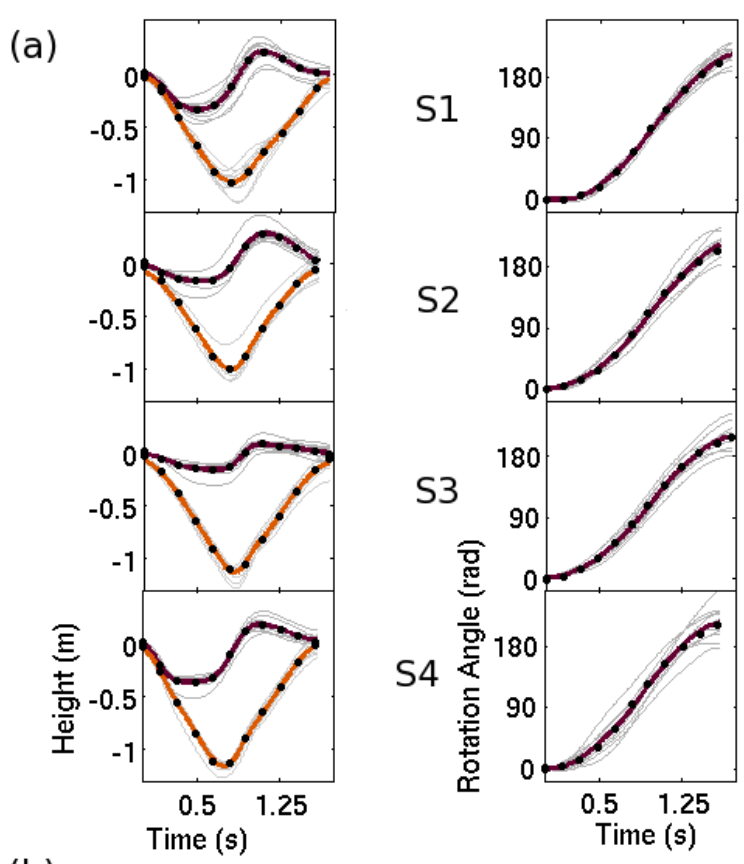

(b)

\begin{tabular}{|l||c|c|c|c||c|}
\hline & S1 & S2 & S3 & S4 & Average \\
\hline$\Theta$ & $3.5 e^{-5}$ & $3.5 e^{-5}$ & $3.5 e^{-5}$ & $3.3 e^{-5}$ & $3.5 e^{-5}$ \\
$\beta$ & 0.86 & 0.83 & 0.9 & 0.76 & 0.84 \\
$\gamma$ & $2.2 e^{-4}$ & $2 e^{-4}$ & $1.5 e^{-4}$ & $1 e^{-5}$ & $1.4 e^{-4}$ \\
$\rho$ & $1.69 e^{-4}$ & $1.74 e^{-4}$ & $1.4 e^{-4}$ & $1.69 e^{-4}$ & $1.63 e^{-4}$ \\
$\Delta h$ & 0.041 & 0.043 & 0.044 & 0.044 & - \\
$l_{\max }$ & 1.017 & 1.009 & 1.032 & 1.015 & - \\
\hline
\end{tabular}

Fig. 3. (a) Match between human experiments and identified model for all four subjects s1 - s4: thin grey lines show 10 measured cycles, black dots their average, and solid lines show computational results. (b) Resulting parameter data for subjects $\mathrm{s} 1-\mathrm{s} 4$, and average values.

conditions. This technique is highly efficient and allows the solution of the described yoyo problem in approximately 1 second.

Such an identification problem is solved individually for each of the four subjects. For each subject we use data of 10 different yoyo cycles out of a nearly cyclic motion, and from this obtain average cyclic data $\hat{x}_{\text {pos }}$. Motion data of subjects can not be combined since every subject has its own characteristic way of playing yoyo, i.e. uses its individual control scheme. However, since all are playing with the same yoyo, we do expect to identify the same yoyo parameters $\Theta$, $\gamma, \beta$ and $\rho$ from the experiments. Parameters $l_{\max }$ and $\Delta_{h}$ again are subject specific, since they depend on the particular way of attaching the yoyo to the hand, and on the marker position on the subjects hand.

Fig. 3-a presents the solutions of the four optimal control problems identifying the cyclic motions of the four subjects with trajectories for hand and yoyo height in the left column, and for the rotational angle in the right column. The thin lines show the 10 different measurements for each subject, while the solid thick lines show the computed trajectories, which nearly perfectly match the average data. It can therefore be concluded that the model results in a very good approximation of the experiments. The table in Fig. 3-b, shows the resulting yoyo parameters for all subjects as well as the average values which can now be used for the following yoyo computations.

\section{GENERATION OF HRP-2 FEASIBLE YOYO MOTION}

The yoyo playing trajectories of human players studied in the previous section cannot directly be transferred to the humanoid robot HRP-2. The required hand motion is not feasible within the tight ranges on position, velocity and acceleration of the robot. We therefore generated new yoyo playing motion which respected the following bounds of HRP-2:

$$
\begin{aligned}
& -0.24 m \leq h \leq 0.16 m \\
& \text { i.e. } \quad 0.94 m-1.34 m \text { above ground } \\
& -1.6 \mathrm{~m} / \mathrm{s} \leq v_{h} \leq 1.6 \mathrm{~m} / \mathrm{s} \\
& -10.5 \mathrm{~m} / \mathrm{s}^{2} \leq a_{h} \leq 10.5
\end{aligned}
$$

as well as the condition that the yoyo should stay well above the floor,

$$
z \geq-1.1 m
$$

HRP-2, at a standing height of $1.54 \mathrm{~m}$ is also shorter than most adults. The yoyo string length was shortened to take this into account. String length was adjusted by following the rule of thumb of measuring a beginner's yoyo string length from below navel height, and we chose $l_{\max }=0.85 \mathrm{~m}$ for the robot.

Fig. 4 shows the geometry of the robot hand and the relative position of the yoyo just before yoyo release (left) and during yoyo playing (right). It follows from the right picture that the offset between hand position and top most yoyo position during the cycle is $\Delta_{h}=0.137 \mathrm{~m}$.

In order to determine a yoyo trajectory suited for the robot, we have again solved an optimal control problem with a similar formulation as above in (24) -(29), but with some differences:

- Equations (30) - (32) have been included in the continuous inequality constraints (29)

- Objective function (24) has been replaced by a function punishing the square of hand accelerations

$$
\min _{x(\cdot), u(\cdot), p, \tau} \int_{0}^{T} a_{h}^{2} d t .
$$

In this formulation, $\tau$, the vector of all phase switching times becomes a free variable of the optimal control problem, since the phase durations are not fixed a priori.

- In order to guarantee continuous acceleration profiles, we have added one more derivative level to the kinematic description, with

$$
\dot{a}_{h}=j_{h}
$$

where the jerk $j_{h}$ now becomes the new control variable of the problem (which is discretized as a piecewise constant function) and $a_{h}$ the eighth state variable (which consequently is piecewise linear and continuous). To 


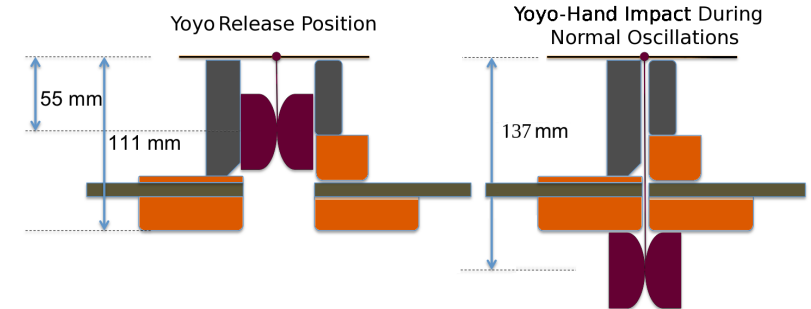

Fig. 4. HRP-2's hand holding the yoyo before release, and in closed position while playing the yoyo

ensure continuity of the accelerations also between cycles, we add to (23) a fifth periodicity constraint

$$
a_{h}(T)=a_{h}(0)
$$

Fig. 5 shows the resulting trajectories for periodic yoyo motions of all eight state variables. All bounds are satisfied, but while velocity and acceleration stay clear of the imposed bound, the possible range of hand height positions is nearly fully exploited. We also computed a special starting cycle that allows us to bring the yoyo from its static release position (compare Fig. 4) in one oscillations to the periodic target orbit.

In order to implement more diverse yoyo playing motions on the humanoid robot, we have also investigated the variations of trajectories with respect to different frequencies, i.e. different cycle times, which can be achieved for the same string length by different hand motion cycle frequencies. In order to determine these different solutions, we repeatedly solve the optimal control problem described above with objective function (34), each time fixing the total time $\tau_{3}=$ $T$ to the chosen value. It is a simple task to additionally provide transition trajectories between the different cyclic trajectories and thus enable the robot to vary between slower and faster yoyo playing. The results for several different cycle times are shown in Fig. 6-a.

We also have investigated the dependency of the robot trajectories on the chosen string length $l_{\max }$. For each of these string lengths, different solutions with different cycle times could be generated; here we have chosen to compare solutions with the optimal cycle times (with respect to criterion (34)) for each string length (see Fig. 6-b).

There are different options on how to implement the solutions computed above on a humanoid robot:

- A fully Open-loop approach: replay the computed hand trajectories while respecting the initial conditions. Due to stability reasons (of the yoyo oscillation) it can be expected that this approach only works for a couple of cycles.

- An Event-based Open-loop strategy using feedback of some key events: a detection of impacts between phases (at the bottom and at the hand) can be used for timing corrections of the executed trajectory (i.e the trajectory is executed phase-wise).This mode corresponds to blindfold yoyo playing of humans, ad it can be expected that the event feed-back already stabilizes
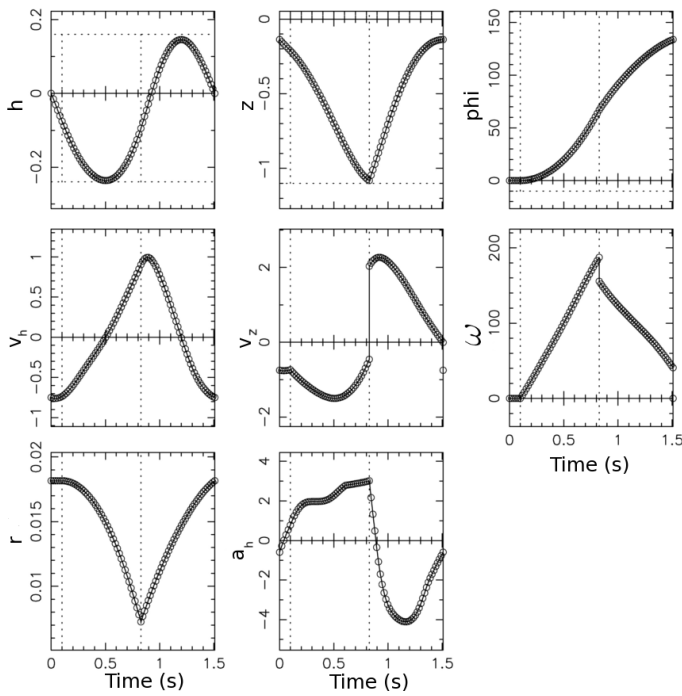

Time (s)

Fig. 5. Optimal smooth yoyo playing trajectory for HRP-2 with continuous hand acceleration profil, respecting bounds on $h, v_{h}$ and $a_{h}$

the yoyo motion significantly. The detecting of phases could be via force feedback from the robot's hand, or internal/external vision information. This mode is also similar to the biologically motivated strategy proposed in [5].

- A Closed-loop approach using vision feedback: yoyo height and/or string length can be constantly tracked using either the robot's own vision system or an external source like a motion capture system. This type of feedback information matches the amount of information that humans may use for yoyo playing.

\section{A. Making the humanoid play}

The humanoid HRP-2, is a 30 DoF robot with complicated redundant kinematic chains and dynamics requirements. While not all $30 \mathrm{DoFs}$ are required to execute the yoyo motion, a majority of the DoFs are also involved in maintaining its balance. For a cyclic motion like playing the yoyo, the humanoid hand is outstretched like a cantilever and moved at close to its velocity limitations. Understandably, the critical need here is to stabilize the robot while managing to execute the generated hand trajectory. To do this we use an implementation of a constraint-based prioritized inverse kinematics solver developed in our laboratory to generate whole-body motion [10]. This implements the approach in [11] to solve kinematic redundancy and packages it with Zero Moment Point (ZMP) based formulation to test for humanoid stability [12]. Using this formulation we computed wholebody motion of the humanoid every $10 \mathrm{~ms}$. A complete description of the dynamics computation and motion constraints is beyond the scope of this paper (for more details, see [10]).

\section{ROBOT EXPERIMENTS}

We implemented the first two strategies for yoyo playing on our humanoid HRP-2, i.e. Open-loop and Eventbased Open-loop. The third strategy, closed-loop control via 


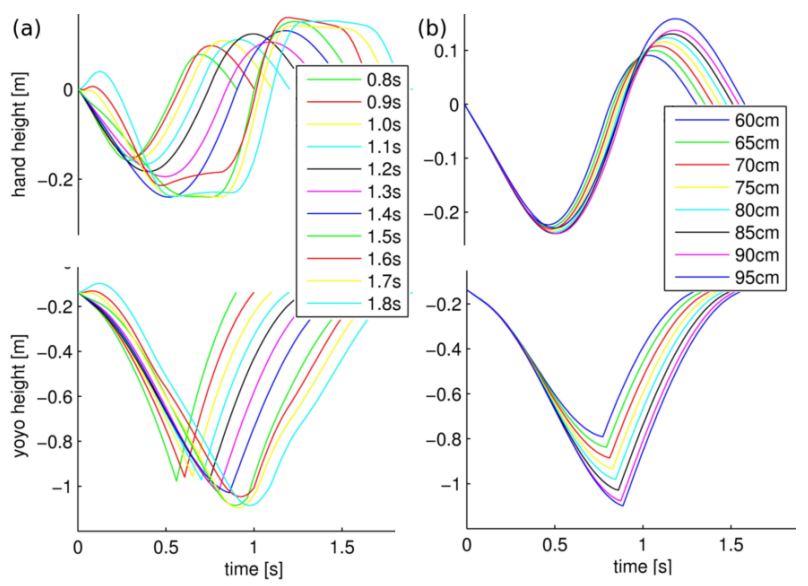

Fig. 6. (a) Cycle time variations of yoyo playing trajectories for HRP-2 (b) Hand and yoyo trajectories for different string lengths $l_{\max }$

vision system, required solving extensive practical issues like latency in tracking the yoyo in real-time, and also the development of a suitable control law based on that vision information. This will be the topic of future work in this direction.

During yoyo playing, humans stabilise the yoyo between oscillations by letting it strike their hand smoothly without introducing any perturbations. Comparatively, HRP-2's hand is an uneven gripper offering no damping whatsoever to a rebounding yoyo. Thus, we first had to solve the problem of hand-yoyo impacts on HRP-2 before implementing any calculated motion. Any solution would also have to take into account that the yoyo would have to be released from the same hand in a controlled manner and with precise timing.

After testing various solutions we settled on the simple construction of two halves of damping sponges attached to the grippers (see Fig. 4). Before release, the yoyo was held between the grippers and suspended from a small inflexible stick attached to the top of the wrist. The wrist is kept horizontal during release, and throughout the oscillation, to cause minimal swinging action in the sagittal plane. After yoyo release, the grippers rapidly close and present the rebounding yoyo with a flat and soft surface on which to impact.

It was practically infeasible to start the hand motion and release the yoyo such that it directly enters the computed periodic orbit. Thus we computed a special release cycle that allowed the transfer - within one yoyo cycle - from a controlled starting position (with zero velocity) onto the desired orbit.

\section{A. Open-loop control}

Simply replaying the computed optimized motion on HRP2, lead to about 6-7 oscillations on average. Fig. 7 shows snapshots of HRP-2 during one oscillation with the most important phases of yoyo and hand motion. Fig. 8 plots the theoretical motion of HRP-2's hand and yoyo, and that actually recorded by motion capture. The yoyo behaved closely to the expected motion in some oscillations, but was different in the others (first 3-4 oscillations). This would be expected because HRP-2 had no perception of the yoyo state, and did not modify its motion to compensate for these differences. However, it should be noted that 6-7 oscillations with a purely open-loop solution is already quite successful and underlines the effectiveness of our model and the optimized trajectory.

\section{B. Event-based switched open-loop control}

To implement event-based control we needed to first reliably detect the important yoyo states, i.e. bottom impact of the yoyo and hand-yoyo impact. HRP-2's hand is equipped with tri-axial force sensors, and we used these sensors to detect the pull of the yoyo string when it is completely stretched.

If the bottom impact occurs before the theoretical impact (as determined by the optimal model), then the humanoid jumps to the trajectory of the rebound phase of the yoyo (and similarly for hand impacts). In some cases, the yoyo would fail to reach the hand and hence no hand impact is registered. In this case the humanoid plays the original trajectory to the end of the cycle, and if still no hand impact is registered, switches to the start of the next cycle. The only way to completely avoid this would be by implementing realtime control using visual information of the yoyo.

The importance of cleanly detecting the bottom impact is paramount in this control approach. In reality we found that HRP-2's force sensors, while sensitive enough to detect these impacts, were also confusing yoyo impact with minor perturbations in the hand motion (due to motor friction, motion stabilizers etc). Consequently, we only managed to get between 3-4 complete oscillations with the unreliable event detection.

Currently, we are working on replacing/supplementing the force sensor event detection by using vision information. The robot's own cameras are not suitable for this task since they have a refresh rate of only $15 \mathrm{~Hz}$. The obvious other choice was the motion capture system which outputs very reliable data at $100 \mathrm{~Hz}$ (which is the same as HRP2's perception loop). But typically there is a transmission delay of 300-400 ms between motion capture and HRP2 's perception loop. Since the total oscillation is about $1600 \mathrm{~ms}$, this is a very significant delay and would need special treatment in the control implementation. The videos of the experiments conducted on HRP-2 can be accessed at http://www.laas.fr/ manu/videos/icra2010Yoyo.mpg

\section{CONCLUSIONS AND FUTURE WORK}

In this paper, we have presented an approach to generate yoyo motions for the humanoid robot HRP-2 based on motion capture data of human yoyo trajectories, a multi-phase model of the yoyo with discontinuities and numerical optimal control techniques. It has been shown that the established yoyo model and an appropriate choice of inputs and model parameters result in very good approximation of experimental data. This model forms the basis for the generation of feasible yoyo motions for HRP-2 respecting its bounds on 


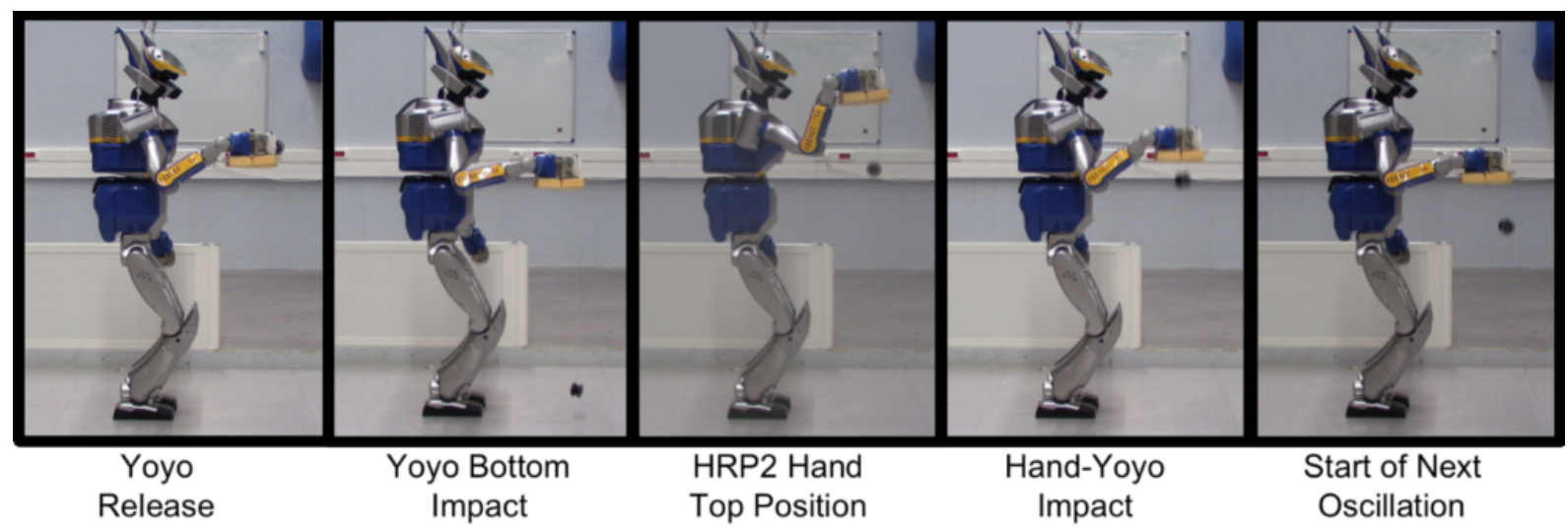

Fig. 7. Snapshots of HRP-2 during the first oscillation of the yoyo. The release phase starts with the yoyo inside the robot's hand, and in consecutive oscillations the yoyo impacts with the damping pad.

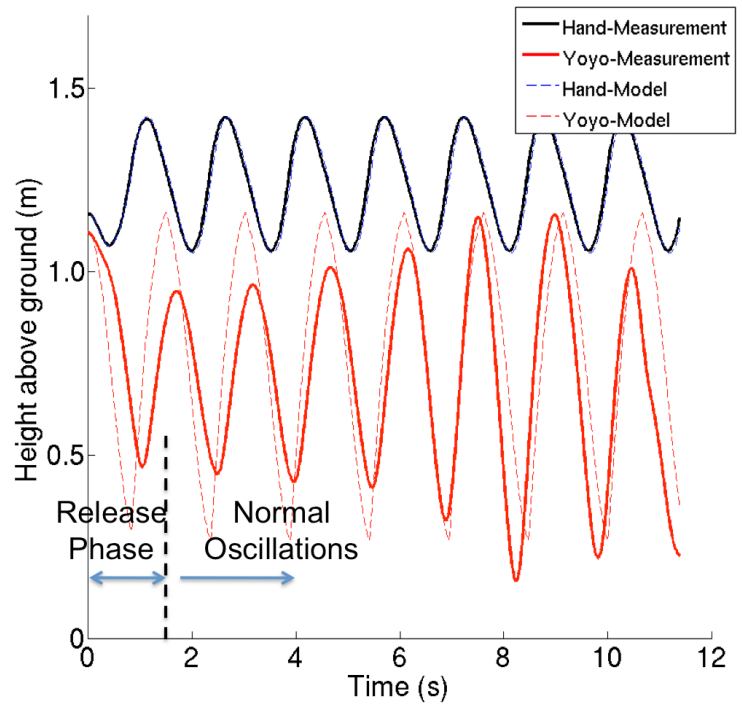

Fig. 8. Open-loop yoyo playing: comparison of computed and real trajectories for hand and yoyo height for 7 successful oscillations (release cycle and 6 periodic cycles)

hand position, velocities and accelerations. Periodic yoyo motions for HRP-2 for different cycle times and string lengths have been generated by solving of optimal control problems. We have started to implement these trajectories on HRP-2 using two different control strategies: a purely openloop control strategy, and an event based switched partly open-loop strategy. Currently these attempts have lead to the successful execution of 7 stable yoyo cycles. The limitation of cycles was not caused by the control strategies used, but rather by practical problems, such as reliable detection of yoyo impacts.

Besides resolving these practical issues and aiming at generating more oscillations with the current control approach, the research will be extended in several directions in the future. We plan to develop biologically inspired closed loop control laws for robotic yoyo playing by evaluating vision-based feedback from the motion capture vision. We also will extend the yoyo model in order to be able to describe more complex yoyo maneuvers in the vertical plane or in 3 dimensions. In addition, the analysis of human yoyo motion will be lead further with the goal to identify objective functions of human yoyo playing by inverse optimal control.

\section{ACKNOWLEDGMENTS}

The authors would like to thank Anthony Mallet for his help with experiments on HRP-2, and Philippe Souères and Jean-Paul Laumond for helpful discussions on the topic. We thank the Simulation \& Optimization group at IWR, University of Heidelberg for providing the optimal control package MUSCOD. We are grateful to the reviewers for their comments and time spent reviewing this work.

\section{REFERENCES}

[1] M. Buehler and D. Koditchek, "From stable to chaotic juggling: theory, simulations and experiments," in Proceedings of ICRA. IEEE RAS, 1990, pp. 1976-1981

[2] S. Schaal and C. G. Atkeson, "Open loop stable control strategies for robot juggling," in IEEE International Conference on Robotics and Automation, 1993, pp. 913 - 918.

[3] G. Bätz, M. Sobotka, D. Wollherr, and M. Buss, "Robot basketball: Ball dribbling - a modified task," in Proceedings of German Workshop on Robotics, 2009.

[4] K. Hashimoto and T. Noritsugu, "Modeling and control of robot yoyo with visual feedback," in Proceedings of ICRA, vol. 3. IEEE RAS, 1996, pp. 2650-2655.

[5] H. L. Jin and M. Zacksenhouse, "Yoyo dynamics: Sequence of collisions captured by a restitution effect," Journal of Dynamical Systems and Control, vol. 124, 390 - 3972002.

[6] H.-L. Jin and M. Zacksenhouse, "Robotic yoyo playing with visual feedback," IEEE Transactions of Robotics, vol. 20, no. 4, pp. 736-744, 2004

[7] L. Zlajpah and B. Nemec, "Control strategy for robotic yoyo," in Proceedings of IROS, vol. 3. IEEE/RSJ, Nov. 2003, pp. 767-772.

[8] H. G. Bock and K.-J. Plitt, "A multiple shooting algorithm for direct solution of optimal control problems," in Proceedings of the 9th IFAC World Congress, Budapest. International Federation of Automatic Control, 1984, pp. 242-247.

[9] D. B. Leineweber, I. Bauer, H. G. Bock, and J. P. Schlöder, "An efficient multiple shooting based reduced SQP strategy for large-scale dynamic process optimization - part I: theoretical aspects," Comput. Chem. Engng, vol. 27, pp. 157 - 166, 2003.

[10] E. Yoshida, O. Kanoun, C. Esteves, and J.-P. Laumond, "Taskdriven support polygon humanoids," in Humanoid Robots, IEEE-RAS International Conference on, 2006.

[11] Y. Nakamura, "Advanced Robotics: Redundancy and Optimization". Boston: Addison-Wesley Longman Publishing, 1991.

[12] M. Vukobratovic and J. Stepanenko, "On the stability of anthropomorphic systems," Mathematical Biosciences, vol. 15, pp. 1-37, 1972. 